\title{
A NOTE ON EXTINCTION TIMES FOR THE GENERAL BIRTH, DEATH AND CATASTROPHE PROCESS
}

\author{
PHIL POLLETT *** AND \\ HANJUN ZHANG, ${ }^{* * * *}$ The University of Queensland \\ BENJAMIN J. CAIRNS, ${ }^{* * * *}$ University of Bristol
}

\begin{abstract}
We consider a birth, death and catastrophe process where the transition rates are allowed to depend on the population size. We obtain an explicit expression for the expected time to extinction, which is valid in all cases where extinction occurs with probability 1 .
\end{abstract}

Keywords: Population process; hitting time; catastrophe; zeta distribution

2000 Mathematics Subject Classification: Primary 60J27

Secondary $60 \mathrm{~J} 35$

The model under consideration is a continuous-time Markov chain $(X(t), t \geq 0)$ taking values in $S=\{0,1, \ldots\}$, where $X(t)$ represents the number in a population at time $t$. When there are $i$ individuals present the population size changes at rate $f_{i}(>0)$, and when a change occurs it is the result of a birth with probability $a(>0)$ or a catastrophe of size $k$ (i.e. the removal of $k$ individuals) with probability $d_{k}(k \geq 1)$. (Simple death events are catastrophes of size 1.) We assume that $d_{k}>0$ for at least one $k \geq 1$ and $a+\sum_{k \geq 1} d_{k}=1$. Thus, the process has transition rates $Q$ given by

$$
q_{i j}= \begin{cases}f_{i} \sum_{k \geq i} d_{k}, & j=0, i \geq 1, \\ f_{i} d_{i-j}, & j=1,2, \ldots, i-1, i \geq 2, \\ -f_{i}, & j=i, i \geq 1, \\ f_{i} a, & j=i+1, i \geq 1, \\ 0, & \text { otherwise, }\end{cases}
$$

and the sole absorbing state 0 , corresponding to population extinction, is accessible from the irreducible class $\{1,2, \ldots\}$. The special case $f_{i}=\rho i$, where $\rho(>0)$ is a per-capita transition rate, was studied by Brockwell [1], Pakes [5], Pakes and Pollett [6], and Pollett [7]. Cairns and Pollett [2] studied the case of general $f_{i}$, providing an explicit expression for the expected time to extinction in the subcritical case. They noted that the supercritcal case could be handled by way of a standard transformation involving the extinction probabilities, and that the critical case

\footnotetext{
Received 22 November 2006.

* Postal address: Department of Mathematics, The University of Queensland, Brisbane, QLD 4072, Australia.

** Email address: pkp@maths.uq.edu.au

*** Email address: hjz@maths.uq.edu.au

**** Postal address: School of Biological Sciences, University of Bristol, Bristol BS8 1UG, UK.

Email address: ben.cairns@bristol.ac.uk
} 
could be handled if the variance of the catastrophe size distribution was finite. The purpose of this note is to point out that this latter condition is not needed.

Let

$$
d(s)=a+\sum_{i=1}^{\infty} d_{i} s^{i+1}
$$

$|s|<1$, be the probability generating function (PGF) of the jump-size distribution. Then the drift $D=1-d^{\prime}(1-)=a-\sum_{i=1}^{\infty} i d_{i}$ satisfies $-\infty \leq D \leq 1$, and the process is said to be subcritical, critical or supercritical according to whether $D<0, D=0$ or $D>0$. Now let $b(s)=d(s)-s$ and $e(s)=1 / b(s)$. It is well known (Section V.12 of Harris [3]) that $e(s)$ has a power series expansion with strictly positive coefficients $\left(e_{i}, i \geq 0\right)$ and with radius of convergence $\sigma$, where $\sigma$ is the smallest zero of $b(s)$ on $(0,1]$. (Indeed this is true for any PGF $d(s)$ with $d(0)>0$.) Furthermore, $\sigma=1$ or $\sigma<1$ according to whether $D \geq 0$ or $D<0$, and $b(s)>0$ for all $s \in[0, \sigma)$. If as we shall assume here $D \leq 0$, then the process is non-explosive and absorption occurs with probability 1 (Pakes [5]). We will prove the following result, which is Theorem 2 of [2] extended to include the $D=0$ case.

Theorem 1. For the subcritical or critical birth, death and catastrophe process, the expected extinction time $\tau_{i}$, starting in state $i$, is finite if and only if $\kappa:=\sum_{i=1}^{\infty} \sigma^{i} / f_{i}<\infty$, in which case $\tau_{0}=0, \tau_{1}=\kappa / a$, and $\tau_{i}=\kappa e_{i-1}-\sum_{j=1}^{i-1} e_{i-1-j} / f_{j}(i \geq 2)$.

Cairns and Pollett's proof [2] for the subcritical case rested on the facts that $e_{i} / e_{i+1} \leq \sigma$ and $e_{i} / e_{i+1} \rightarrow \sigma$, which they deduced from results obtained by Yang [8] concerning the invariant measure of a Markov branching process. However, they could be applied to the birth, death and catastrophe process only in the $D<0$ case. Lemma 1 shows that the two conditions hold whatever the sign of $D$. Thus, Theorem 1 is established.

Lemma 1. Let $p(s)$ be a PGF with $p(0)>0$ and let $\sigma$ be the smallest zero of $p(s)$ on $(0,1]$. Then, the coefficients $\left(e_{i}, i \geq 0\right)$ of the power series $e(s)=1 /(p(s)-s)$ satisfy

(i) $\sigma^{-i} \leq e_{i} \leq \sigma e_{i+1}$, and

(ii) $\lim _{i \rightarrow \infty} e_{i} / e_{i+1}=\sigma$.

Proof. Let $m(s)=\sum_{i=0}^{\infty} m_{i} s^{i}$ be a PGF with $m(0)>0$ and $m^{\prime}(1-) \leq 1$, so that 1 is the smallest root of $m(s)=s$ on $[0,1]$. As already noted, $h(s)=1 /(m(s)-s)$ is a power series with nonnegative coefficients $\left(h_{i}, i \geq 0\right)$. We will prove that $1 \leq h_{i} \leq h_{i+1}(i \geq 0)$ and that $h_{i} / h_{i+1} \rightarrow 1$. The proof of the lemma will then follow by taking $m_{1}=p_{1} \sigma+1-\sigma$ and $m_{i}=p_{i} \sigma^{i}(i \neq 1)$.

Following Harris (Section V.12 of [3]), we write

$$
\frac{1}{m(s)-s}=\frac{1}{(1-s)(1-\Psi(s))},
$$

where $\Psi(s)=(1-m(s)) /(1-s)$, which is analytic at $s=0$ and has the following power series expansion with nonnegative coefficients: $\Psi(s)=1-m_{0}+\left(1-m_{0}-m_{1}\right) s+\left(1-m_{0}-\right.$ $\left.m_{1}-m_{2}\right) s^{2}+\cdots$. Setting

$$
\sum_{i=0}^{\infty} g_{i} s^{i}=\frac{1}{1-\Psi(s)}=1+\Psi(s)+\Psi(s)^{2}+\Psi(s)^{3}+\cdots,
$$


we see that $g_{0} \geq 1$ and $g_{i} \geq 0(i \geq 1)$. From (1) and (2) we have

$$
\sum_{i=0}^{\infty} h_{i} s^{i}=\frac{1}{m(s)-s}=\left(1+s+s^{2}+s^{3}+\cdots\right) \sum_{i=0}^{\infty} g_{i} s^{i} .
$$

Hence, $h_{i}=\sum_{j=0}^{i} g_{j}$, and so $h_{i+1} \geq h_{i} \geq 1$.

Next, since

$$
\sum_{i=0}^{\infty} g_{i} s^{i}=\frac{1}{1-\Psi(s)}=\frac{\Psi(s)}{1-\Psi(s)}+1,
$$

we have $g_{0}=\psi_{0} g_{0}+1$ and $g_{i}=\sum_{j=0}^{i} \psi_{j} g_{i-j}(i \geq 1)$, where $\psi_{i}=1-m_{0}-m_{1}-\cdots-m_{i}$. It follows that $g_{0}=1 /\left(1-\psi_{0}\right)$ and $g_{i}=g_{0} \sum_{j=1}^{i} \psi_{j} g_{i-j}(i \geq 1)$. We will prove that $g_{i} \leq g_{0}$ ( $i \geq 0$ ) using mathematical induction. The statement is clearly true for $i=0$. So, suppose that, for some fixed $i \geq 1, g_{j} \leq g_{0}$ for all $j \leq i-1$. Then,

$$
g_{i}=\frac{1}{1-\psi_{0}} \sum_{j=1}^{i} \psi_{j} g_{i-j} \leq \frac{g_{0}}{1-\psi_{0}} \sum_{j=1}^{i} \psi_{j} \leq g_{0},
$$

where in the last inequality we have used the fact that $\sum_{j=0}^{\infty} \psi_{j}=m^{\prime}(1-) \leq 1$.

As $h_{i}=\sum_{j=0}^{i} g_{j}$, we have $1 \leq h_{i+1} / h_{i}=1+g_{i+1} / h_{i}$. Also, as $\left(h_{i}\right)$ is an increasing sequence, the limit $L=\lim _{i \rightarrow \infty} h_{i}$ exists, but might be infinite. If $L<\infty$, then $g_{i} \rightarrow 0$, and so $g_{i+1} / h_{i} \rightarrow 0$. Otherwise, if $L=\infty$, then $g_{i+1} / h_{i} \rightarrow 0$, because $g_{i} \leq g_{0}$. In either case we have $h_{i+1} / h_{i} \rightarrow 1$.

On setting $m_{1}=p_{1} \sigma+1-\sigma$ and $m_{i}=p_{i} \sigma^{i}(i \neq 1)$, it is clear that $m_{0}=p_{0}>0$, $\sum_{i=0}^{\infty} m_{i}=1$, and $m(s)=\sum_{i=0}^{\infty} p_{i} \sigma^{i} s^{i}+(1-\sigma) s$. Hence, $m(s)-s=p(\sigma s)-\sigma s$, and so $h_{i}=e_{i} \sigma^{i}$ and 1 is the smallest root of $m(s)=s$ on $[0,1]$ because $\sigma$ is the smallest root of $p(s)=s$ on $[0,1]$. The first claim, that $\sigma^{-i} \leq e_{i} \leq \sigma e_{i+1}(i \geq 0)$, now follows because $1 \leq$ $h_{i} \leq h_{i+1}$. To prove the second claim, observe that $\lim _{i \rightarrow \infty} e_{i} / e_{i+1}=\sigma \lim _{i \rightarrow \infty} h_{i} / h_{i+1}=$ $\sigma$. The proof is complete.

Example. We will suppose that catastrophe sizes follow the zeta distribution (also known as the Zipf or 'discrete Pareto' distribution), an example of a power law that has been used to model the effect of a variety of hazards, such as the area burnt in forest fires [4]. We set $d_{k}=(1-a) k^{-r} / \zeta(r)$, where $\zeta(\cdot)$ is the Riemann zeta function and $r$ is a parameter that determines the weight ascribed to the tail of the distribution. Then, $b(s)=a-s+(1-a)$ $\times s \operatorname{Li}_{r}(s) / \zeta(r)$, where $\operatorname{Li}_{r}(\cdot)$ is the (real-valued) polylogarithm function of order $r$. The distribution $\left(d_{k}, k \geq 1\right)$ is only defined for $r>1$ and its mean is finite only if $r>2$; for $1<r \leq 2, D=-\infty$, while for $r>2, D$ is finite and its value depends on $a$. The variance of the distribution is finite if and only if $r>3$, and so when $2<r \leq 3$ the mean is finite but the variance is infinite. For example, when $r=3$, it can be shown that $D<0, D=0$ or $D \geq 0$, according to whether $a$ is less than, equal to, or greater than $\pi^{2} /\left[6 \zeta(3)+\pi^{2}\right]$. The expected extinction times $\left(\tau_{i}, i \geq 0\right)$ may then be obtained from Theorem 1. If in particular $D=0$, then $\sigma=1$ and thus, the $\tau_{i}$ are finite if and only if $\kappa=\sum_{i=1}^{\infty} 1 / f_{i}$ is finite (i.e. if changes in the population size occur 'fast enough' as the population size increases). For the linear case $f_{i}=\rho i$, the birth, death and catastrophe process with zeta catastrophes and $r=3$ has finite or infinite expected extinction times according to whether $D<0$ or $D \geq 0$. Alternatively, if $f_{i}=\rho i(i+1)$ then the expected extinction times are finite when $D \leq 0$; when $D>0$ the process is explosive (Theorem 1 of [2]). 


\section{Acknowledgement}

The authors acknowledge the support of the Australian Research Council Centre of Excellence for Mathematics and Statistics of Complex Systems.

\section{References}

[1] Brockwell, P. (1985). The extinction time of a birth, death and catastrophe process and of a related diffusion model. Adv. Appl. Prob. 17, 42-52.

[2] Cairns, B. And Pollett, P. (2004). Extinction times for a general birth, death and catastrophe process. J. Appl. Prob. 41, 1211-1218.

[3] Harris, T. (1963). The Theory of Branching Processes. Springer, Berlin.

[4] Malamud, B. D., Morein, G. And Turcotte, D. L. (1998). Forest fires: an example of self-organized critical behaviour. Science 281, 1840-1842.

[5] Pakes, A. G. (1987). Limit theorems for the population size of a birth and death process allowing catastrophes. J. Math. Biol. 25, 307-325.

[6] Pakes, A. G. AND Pollett, P. K. (1989). The supercritical birth, death and catastrophe process: limit theorems on the set of extinction. Stoch. Process. Appl. 32, 161-170.

[7] Pollett, P. (2001). Quasi-stationarity in populations that are subject to large-scale mortality or emigration. Environ. Internat. 27, 231-236.

[8] Yang, Y. (1973). Asymptotic properties of the stationary measure of a Markov branching process. J. Appl. Prob. 10, 447-450. 